\title{
HIGHLIGHTS
}

BONE

\section{Hyponatremia-induced osteoporosis}

Hyponatremia (defined as serum sodium concentration $<135 \mathrm{mmol} / \mathrm{l}$ ) represents a previously unrecognized cause of osteoporosis, say researchers of a new study published in the Journal of Bone and Mineral Research.

"A clinical case, in which severe osteoporosis was found in a patient with hyponatremia due to the syndrome of inappropriate antidiuretic hormone secretion (SIADH), without any demonstrable etiology for the bone loss, raised the possibility of an association between low sodium levels and osteoporosis," recount lead authors Joseph G. Verbalis and Julia Barsony from Georgetown University, Washington DC, USA.

Confirmation of the relationship between low sodium levels and osteoporosis by clinical research methods was, however, frustrated by the presence of multiple comorbidities in patients with SIADH. The investigators turned to animal studies in order to overcome the limitations associated with the evaluation of findings in hyponatremic patients.

Verbalis et al. induced chronic hyponatremia in rats fed a liquid diet by administration of the vasopressin V2 receptor agonist desmopressin for a period of 3 months. Hyponatremia was associated with a $20-30 \%$ reduction in BMD compared with normonatremic rats, a degree of loss that was equivalent to or greater than that seen with any previously reported animal model of osteoporosis. The bone loss in hyponatremic rats was accompanied by a fivefold increase in the number of osteoclasts per bone area, a finding which suggests a resorptive type of osteoporosis.

\section{$\mathbf{4}$....it represents an attempt} of the body to preserve sodium homeostasis at the expense of bone structural integrity... 77

In order to relate their findings to human biology, the researchers used an epidemiological approach and analyzed data from the NHANES (National Health and Nutrition Examination Survey) III study, which includes information on sodium concentrations and BMD of the hip in a nationally representative sample of US adults. The analysis of this cross-sectional cohort showed that the odds ratio of osteoporosis at the hip was significantly increased in patients with hyponatremia aged $\geq 50$ years compared with the general population. "Mechanistically, hyponatremia-induced bone resorption and osteoporosis is unique in that it represents an attempt of the body to preserve sodium homeostasis at the expense of bone structural integrity," comments Verbalis.
The group's ongoing studies focus on the effects that age, sex, severity of hyponatremia and hormonal replacement have on the bone loss induced by hyponatremia. Verbalis and co-workers plan to further "examine the reversibility of the bone loss with correction of hyponatremia and the ability of antiresorptive agents to treat hyponatremia-induced osteoporosis." Furthermore, the researchers hope to elucidate in vitro whether a decreased extracellular sodium concentration represents the signal to mobilize stored sodium from bone via matrix resorption.

"Cellular and molecular studies may lead to the identification of novel osteoclast sensing and signaling mechanisms in response to low extracellular sodium concentrations and potential new drug targets for inhibiting osteoclast-mediated bone resorption," comments Barsony.

In conclusion, although the incidence of osteoporosis as a result of hyponatremia is potentially very low, even mild chronic hyponatremia might act additively or synergistically with other causes of bone loss associated with aging to contribute to morbidity and mortality in the elderly population. The investigators, therefore, suggest that "bone quality should be assessed in all patients with chronic hyponatremia and appropriate treatment initiated where indicated."

Linda Koch

Original article Verbalis, J. G. et al. Hyponatremiainduced osteoporosis. J. Bone Miner. Res. doi:10.1359/ jbmr.090827 